\title{
A Prospective Study of Polyunsaturated Fatty Acid Levels in Blood and Prostate Cancer Risk
}

\section{Citation}

Chavarro, J. E., M. J. Stampfer, H. Li, H. Campos, T. Kurth, and J. Ma. 2007. “A Prospective Study of Polyunsaturated Fatty Acid Levels in Blood and Prostate Cancer Risk." Cancer Epidemiology Biomarkers \&amp; Prevention 16 (7): 1364-70. https://doi.org/10.1158/1055-9965.epi-06-1033.

\section{Permanent link}

http://nrs.harvard.edu/urn-3:HUL.InstRepos:41292893

\section{Terms of Use}

This article was downloaded from Harvard University's DASH repository, WARNING: This file should NOT have been available for downloading from Harvard University's DASH repository.

\section{Share Your Story}

The Harvard community has made this article openly available.

Please share how this access benefits you. Submit a story.

\section{Accessibility}




\title{
A Prospective Study of Polyunsaturated Fatty Acid Levels in Blood and Prostate Cancer Risk
}

\author{
Jorge E. Chavarro, ${ }^{1}$ Meir J. Stampfer, ${ }^{1,2,3}$ Haojie Li, ${ }^{3}$ Hannia Campos, ${ }^{1}$ \\ Tobias Kurth, ${ }^{2,4}$ and Jing $\mathrm{Ma}^{3}$ \\ Departments of ${ }^{1}$ Nutrition and ${ }^{2}$ Epidemiology, Harvard School of Public Health and ${ }^{3}$ The Channing Laboratory and \\ ${ }^{4}$ Divisions of Aging and Preventive Medicine, Department of Medicine, Brigham and Women's Hospital, \\ Harvard Medical School, Boston, Massachusetts
}

\begin{abstract}
Background: Animal models suggest that n-3 fatty acids inhibit prostate cancer proliferation, whereas n-6 fatty acids promote it, but epidemiologic studies do not uniformly support these findings.

Methods: A nested case-control study was conducted among 14,916 apparently healthy men who provided blood samples in 1982. Blood fatty acid levels were determined for 476 men diagnosed with prostate cancer during a 13-year follow-up and their matched controls. Conditional logistic regression was used to estimate the relative risks (RR) and $95 \%$ confidence intervals (95\% CI) of total, non-aggressive (stage A/B and Gleason $<7$ ) and aggressive (stage C/D, Gleason $\geq 7$, subsequent distant metastasis or death) prostate cancer associated with blood levels of specific fatty acids expressed as percentages of total fatty acids.

Results: Whole blood levels of all long-chain n-3 fatty acids examined and of linoleic acid were inversely related to

overall prostate cancer risk $\left(\mathrm{RR}_{\mathrm{Q} 5 \mathrm{vs.} . \mathrm{Q1}}, 0.59 ; 95 \% \mathrm{CI}, 0.38-0.93\right.$; $P_{\text {trend }}=0.01$ for total long-chain n-3 fatty acids and $R R_{Q 5 v s . Q 1}$, $0.62 ; 95 \%$ CI, $0.41-0.95 ; P_{\text {trend }}=0.03$ for linoleic). Blood levels of $\gamma$-linolenic and dihomo- $\gamma$-linolenic acids, fatty acids resulting from the metabolism of linoleic acid, were directly associated with prostate cancer (RR, 1.41; 95\% CI, 0.94-2.12; $P_{\text {trend }}=0.05$ for $\gamma$-linolenic and RR, 1.54; 95\% CI, 1.03-2.30; $P_{\text {trend }}=0.02$ for dihomo- $\gamma$-linolenic acid). Levels of arachidonic and $\alpha$-linolenic acids were unrelated to prostate cancer.

Conclusions: Higher blood levels of long-chain n-3 fatty acids, mainly found in marine foods, and of linoleic acid, mainly found in non-hydrogenated vegetable oils, are associated with a reduced risk of prostate cancer. The direct associations of linoleic acid metabolites with prostate cancer risk deserve further investigation. (Cancer Epidemiol Biomarkers Prev 2007;16(7):1364-70)
\end{abstract}

\section{Introduction}

Prostate cancer is the most frequently diagnosed and second most lethal malignancy among men in the United States (1). Despite its high frequency, few potentially modifiable risk factors have been identified (2), and their etiologic relevance is still debated. Intake of dietary fatty acids is one of these potentially modifiable factors. In vitro studies suggest that n-6 polyunsaturated fatty acids (PUFA) stimulate prostate cancer growth, whereas n-3 fatty acids inhibit it (3). However, epidemiologic studies have not uniformly supported these findings, and some have reported significant associations in the opposite direction suggested by animal and in vitro studies (4). Thus, whether intake of n-3 and n-6 PUFA affects prostate carcinogenesis in humans remains unclear.

In 1994, we reported the association between plasma levels of fatty acids and prostate cancer risk among the participants of the Physician's Health Study (5). This report was based on 6 years of follow-up and included 120 incident prostate cancer cases. In that study, $\alpha$-linolenic acid (18:3n-3) was the only fatty acid that was associated with an increased risk of prostate cancer. Due to the small number of cases, it was not possible to explore this association separately according to tumor characteristics. In addition, only four major PUFA were determined in the plasma samples. Here, we present analyses based on 476

Received 12/12/06; revised 3/20/07; accepted 4/18/07

Grant support: Research grants CA42182, CA58684, and CA90598 and training grant T32 DK-007703.

The costs of publication of this article were defrayed in part by the payment of page charges This article must therefore be hereby marked advertisement in accordance with 18 U.S.C. Section 1734 solely to indicate this fact.

Requests for reprints: Jorge E. Chavarro, Department of Nutrition, Harvard School of Public Health, 665 Huntington Avenue, Boston, MA 02115. Phone: 617-432-4584; Fax: 671-432-2435. E-mail: jchavarr@hsph.harvard.edu

Copyright (C) 2007 American Association for Cancer Research. doi:10.1158/1055-9965.EPI-06-1033 incident cases accrued during 13 years of follow-up of this cohort and explore in detail the association between whole blood levels of different n-3 and n- 6 PUFA and prostate cancer risk.

\section{Materials and Methods}

Study Population. The Physician's Health Study was a randomized, double-blind, placebo-controlled trial evaluating the effect of aspirin and $\beta$-carotene in the primary prevention of heart disease and cancer among 22,071 U.S. male physicians, aged 40 to 84 years in 1982. Men were excluded from the study if they had a history of myocardial infraction, stroke, transient ischemic attack, unstable angina, cancer (except non-melanoma skin cancer), renal or liver disease, peptic ulcer, gout, had a contraindication to use aspirin or were users of aspirin, platelet-active medications, or vitamin A supplements. The aspirin component of the trial was terminated early in 1988 due to the benefits of aspirin on myocardial infraction (6). The $\beta$-carotene component of the trial was terminated in 1995 (7). Written informed consent was obtained from each participant, and the study was approved by the Human Research Committee at Brigham and Women's Hospital.

Participants completed two mailed questionnaires before randomization, where they provided information on several lifestyle factors. Follow-up questionnaires were mailed at 6 and 12 months after randomization and yearly thereafter. In the 6- and 12-month questionnaires, participants completed abbreviated food frequency questionnaires with 19 and 16 nonoverlapping food items, respectively. These data are insufficient to estimate total caloric intake. Pre-randomization blood specimens were obtained from 14,916 (68\%) participants and stored at $-82^{\circ} \mathrm{C}(5)$. The current report is restricted to newly diagnosed prostate cancer cases during the first 13 years of 
follow-up among the men who provided a baseline blood sample. During this period, follow-up was more than $99 \%$ complete for morbidity and $100 \%$ for mortality.

Selection of Cases and Controls. Whenever a participant reported a diagnosis of prostate cancer, we requested hospital records and pathology reports for review by study physicians from the End Points Committee. Using the risk-set sampling method, we selected a control subject for each confirmed case among the men who had provided a blood sample, had not had a partial or total prostatectomy, and had not reported prostate cancer at the time of case diagnosis. Controls were individually matched to cases by age (within 1 year for men aged 55 years or younger and within 5 years for men older than 55 years) and smoking status at baseline (current, former or never). Of the 758 cases accrued through 1995, 505 had enough blood for fatty acid levels determination. Case and control subjects whose blood sample was received $\geq 6$ days after it was drawn were excluded from analyses, leaving 476 cases and their matched controls.

Medical Record Review. Medical records and pathology reports were reviewed by study physicians to determine the tumor stage and Gleason score at diagnosis. Stage was recorded according to the modified Whitemore-Jewett classification scheme (8). Cases without pathologic staging were classified as indeterminate stage unless there was clinical evidence of distant metastases. We classified cases according to their clinical aggressiveness. Non-aggressive cases were defined as those with localized tumors (stage A or B) and Gleason $<7$ at diagnosis. Cases were considered aggressive when they presented as advanced disease (stage $C$ or D) or Gleason $\geq 7$ at diagnosis or subsequently developed distant metastases or died from prostate cancer.

Laboratory Analyses. Blinded samples from cases and their matched controls were processed and analyzed together to reduce any effect of inter-assay variability. Fatty acids were extracted from whole blood into isopropanol and hexane containing $50 \mathrm{mg}$ of 2.6-di-tert-butyl- $p$-cresol as an antioxidant. Fatty acids were transmethylated with methanol and sulfuric acid, as previously described (9-11). After esterification, the samples were evaporated, and the fatty acids were redissolved in iso-octane and quantified by gas-liquid chromatography on a fused silica capillary cis/trans column (SP2560, Supelco). Peak retention times were identified by injecting known standards (NuCheck Prep) and analyzed with the ChemStation A.08.03 software (Agilent Technologies). The fatty acid levels in each sample were expressed as the percentage of total fatty acids. Although we could not directly assess whether long-term storage and freezing affected the fatty acid measurements, n-3 and n-6 PUFA have moderately high reliability coefficients (0.66 and 0.53 , respectively) and minimal oxidation in serum samples stored for up to 12 years at $-80^{\circ} \mathrm{C}(12)$. Coefficients of variation for all fatty acid peaks were measured by analyzing quality control samples (indistinguishable from other study samples) randomly distributed throughout the study samples. The coefficients of variation for the fatty acids

Table 1. Baseline characteristics of prostate cancer cases and control subjects

\begin{tabular}{|c|c|c|c|}
\hline & Cases $(n=476)$ & Controls $(n=476)$ & $P^{*}$ \\
\hline Age, $y^{\dagger}$ & $58[53-64]$ & $58[53-63]$ & Matched \\
\hline Length of follow-up, $y^{\dagger}$ & $9[7-11]$ & - & \\
\hline \multicolumn{4}{|l|}{ Disease status at diagnosis, $\%$} \\
\hline Localized (stage A or B) & 61 & - & \\
\hline Advanced (stage C or D) & 23 & - & \\
\hline Undetermined & 16 & - & \\
\hline \multicolumn{4}{|l|}{ Tumor grade at diagnosis, $\%$} \\
\hline Gleason $<7$ & 51 & - & \\
\hline Gleason $\geq 7$ & 27 & - & \\
\hline Undetermined & 22 & - & \\
\hline \multicolumn{4}{|l|}{ Date of diagnosis, $\%$} \\
\hline Before October 1, 1990 & 33 & - & \\
\hline On or after October 1, 1990 & 67 & - & \\
\hline Smoking status, $\%$ & & & Matched \\
\hline Current & 8 & 8 & \\
\hline Former & 42 & 42 & \\
\hline White/Caucasian, \% & 95 & 93 & 0.49 \\
\hline Height, $\mathrm{m}^{\dagger}$ & $1.78[1.75-1.83]$ & $1.78[1.73-1.83]$ & 0.14 \\
\hline Body mass index, $\mathrm{kg} / \mathrm{m}^{2 \dagger}$ & $24.4[23.1-25.8]$ & $24.2[22.8-25.8]$ & 0.15 \\
\hline Regular multivitamin use, \% & 21 & 24 & 0.45 \\
\hline Vigorous exercise twice per week or more, \% & 58 & 55 & 0.40 \\
\hline Alcohol use once per day or more, $\%$ & 32 & 30 & 0.48 \\
\hline Whole milk once per day or more, $\%$ & 7 & 8 & 0.71 \\
\hline Low-fat milk once per day or more, $\%$ & 27 & 23 & 0.15 \\
\hline Treatment assignment & & & 0.40 \\
\hline Placebo alone & 23 & 28 & \\
\hline Aspirin alone & 26 & 26 & \\
\hline$\beta$-Carotene alone & 26 & 25 & \\
\hline Aspirin and $\beta$-carotene & 25 & 22 & \\
\hline \multicolumn{4}{|l|}{ Blood fatty acids, $\%$ total fatty acids ${ }^{\dagger}$} \\
\hline Linoleic $(18: 2 n-6)$ & $24.9[22.7-26.9]$ & $25.3[23.3-27.2]$ & 0.04 \\
\hline$\gamma$-Linolenic $(18: 3 n-6)$ & $0.27[0.21-0.35]$ & $0.26[0.21-0.33]$ & 0.09 \\
\hline Dihomo- $\gamma$-linolenic $(20: 3 n-6)$ & $1.40[1.24-1.61]$ & $1.36[1.22-1.53]$ & 0.02 \\
\hline Arachidonic (20:4n-6) & $10.2[9.01-11.2]$ & 10.1 [9.00-11.2] & 0.80 \\
\hline$\alpha$-Linolenic $(18: 3 n-3)$ & $0.36[0.29-0.45]$ & $0.35[0.29-0.43]$ & 0.30 \\
\hline Eicosapentaenoic (20:5n-3) & $1.74[1.48-2.01]$ & $1.79[1.52-2.06]$ & 0.06 \\
\hline Docosapentaenoic (22:5n-3) & $0.94[0.84-1.06]$ & $0.97[0.86-1.09]$ & 0.01 \\
\hline Docosahexaenoic $(22: 6 n-3)$ & $2.18[1.81-2.61]$ & $2.19[1.82-2.78]$ & 0.26 \\
\hline Long-chain n-3 & $4.86[4.25-5.51]$ & $4.96[4.30-5.74]$ & 0.06 \\
\hline
\end{tabular}

* $P$ values were computed using the Wilcoxon rank-sum test for continuous variables and the $\chi^{2}$ test for categorical variables.

†Values expressed as median [25th-75th percentile]. 
Table 2. Spearman correlation coefficients between levels of whole blood n-3 and n-6 fatty acids among the controls $(n=476)$

\begin{tabular}{|c|c|c|c|c|c|c|c|c|}
\hline & $18: 3 n-6$ & $20: 3 n-6$ & $20: 4 n-6$ & $18: 3 n-3$ & $20: 5 n-3$ & $22: 5 n-3$ & $22: 6 n-3$ & Trans fats \\
\hline $18: 2 n-6$ & -0.33 & -0.14 & -0.15 & 0.23 & -0.08 & -0.10 & 0.08 & 0.01 \\
\hline $18: 3 n-6$ & & 0.26 & 0.23 & 0.01 & -0.08 & -0.09 & -0.22 & -0.12 \\
\hline $20: 3 n-6$ & & & 0.01 & -0.19 & 0.04 & 0.07 & -0.12 & -0.09 \\
\hline $20: 4 n-6$ & & & & -0.46 & 0.32 & 0.44 & 0.23 & -0.38 \\
\hline $18: 3 n-3$ & & & & & -0.21 & -0.21 & -0.08 & 0.21 \\
\hline $20: 5 n-3$ & & & & & & 0.63 & 0.60 & -0.36 \\
\hline $22: 5 n-3$ & & & & & & & 0.50 & -0.29 \\
\hline $22: 6 n-3$ & & & & & & & & -0.32 \\
\hline
\end{tabular}

NOTE: With this sample size, $P<0.05$ for all $r \geq 10.13 \mid$.

of interest were $2.12 \%$ for linoleic acid $(18: 2 n-6), 3.58 \%$ for $\gamma$-linolenic acid (18:3n-6), $0.95 \%$ for dihomo- $\gamma$-linolenic acid (20:3n-6), $1.70 \%$ for arachidonic acid (20:4n-6), $2.99 \%$ for $\alpha$-linolenic acid (18:3n-3), $6.50 \%$ for eicosapentaenoic acid (20:5n-3), 3.30\% for docosapentaenoic acid (22:5n-3), and 2.65\% for docosahexaenoic acid (22:6n-3). For each participant, we took the sum of the peaks for $20: 5 n-3,22: 5 n-3$, and $22: 6 n-3$ to estimate the total long-chain n-3 fatty acid level. In addition, we divided each participant's blood levels of 18:2n-6 by their $18: 3 n-3$ levels and the levels of $20: 4 n-6$ by those of $20: 5 n-3$ to estimate the ratio of n-6 to n-3 fatty acids of the same carbon chain length.

Statistical Analyses. We calculated median values and proportions of the baseline characteristics of case and control subjects. To evaluate whether these characteristics differed between cases and controls, categorical variables were tested using the $\chi^{2}$ test, and continuous variables were tested using the Wilcoxon rank-sum test. To characterize the associations between the blood levels of different PUFA, expressed as percentage of total fatty acids, we calculated Spearman rankcorrelation coefficients between these fatty acids among the controls.

To estimate the association between blood levels of PUFA and prostate cancer, we first divided cases and controls into five groups according to quintiles of fatty acid levels among the controls. Then, we used logistic regression models conditioned on the matching variables to estimate the relative risk (RR) of prostate cancer in a given quintile of fatty acid level in relation to the lowest quintile. We considered the potential confounding effects of baseline characteristics by adding to the initial model terms for variables associated with prostate cancer and fatty acid levels at $P<0.20$ and evaluating whether adding these variables changed the initial fatty acid estimates by more than $10 \%$. None of the variables evaluated (height, body mass index, and intake of low-fat milk) changed the initial fatty acids estimates substantially; therefore, further adjustment for these variables was not done. Given that all of the $n-6$ fatty acids evaluated can be metabolically derived from 18:2n-6, and all the n-3 fatty acids can be derived from 18:3n-3, and that trans fats share common food sources with 18:2n-6 and 18:3n-3 and can inhibit the enzymes in the metabolism of these fatty acids, we did additional analysis controlling for the blood levels of these three fatty acids.

We assessed the possibility that the association between 18:3n-3 levels and prostate cancer might differ by levels of 18:2n- 6 by introducing cross-product terms between 18:3n-3 levels, as a linear term, and an indicator of low (lowest tertile) 18:2n-6 blood levels. We similarly assessed the possibility that the association between 20:4n-6 and prostate cancer would be modified by $20: 5 n-3$, and that the association of long-chain PUFA would be modified by randomized aspirin assignment given that aspirin affects the cyclooxygenase metabolism of these fatty acids. Lastly, we refitted the regression models in subgroups defined by tumor stage, grade, aggressiveness, and by diagnosis before (through September 30, 1990) or after (since October 1, 1990) the widespread use of prostate-specific antigen screening. Tests for linear trend were conducted in all models by using the median fatty acid levels in each quintile as a continuous variable. All statistical analyses were done using SAS, version 9.1 (SAS Institute). Results were considered to be statistically significant when $P<0.05$ (two tailed).

\section{Results}

At baseline, subjects who later developed prostate cancer had higher blood levels of 20:3n-6 than controls, whereas controls had higher blood levels of 18:2n-6, 20:5n-3, and 22:5n-3 (Table 1). The blood levels of 20:4n-6, 20:5n-3, and 22:5n-3 were slightly higher among men who provided fasting samples ( $\geq 8 \mathrm{~h}$ since last meal) than for men with non-fasting samples. The median blood levels for non-fasting versus fasting samples were 9.98 and 10.5 for 20:4n-6, 1.74 and 1.87 for $20: 5 n-3$, and 0.94 and 0.99 for $22: 5 n-3$. There were no significant differences in the blood levels of the remaining fatty acids according to fasting status or for any fatty acid according to the time since blood draw and receipt of the blood sample.

There were multiple correlations (Table 2) between the blood levels of individual PUFA (Fig. 1). Among n-6 fatty acids, 18:2n-6 levels were inversely related to the levels of fatty acids resulting from its desaturation and elongation; levels of
N-6 FATTY ACIDS

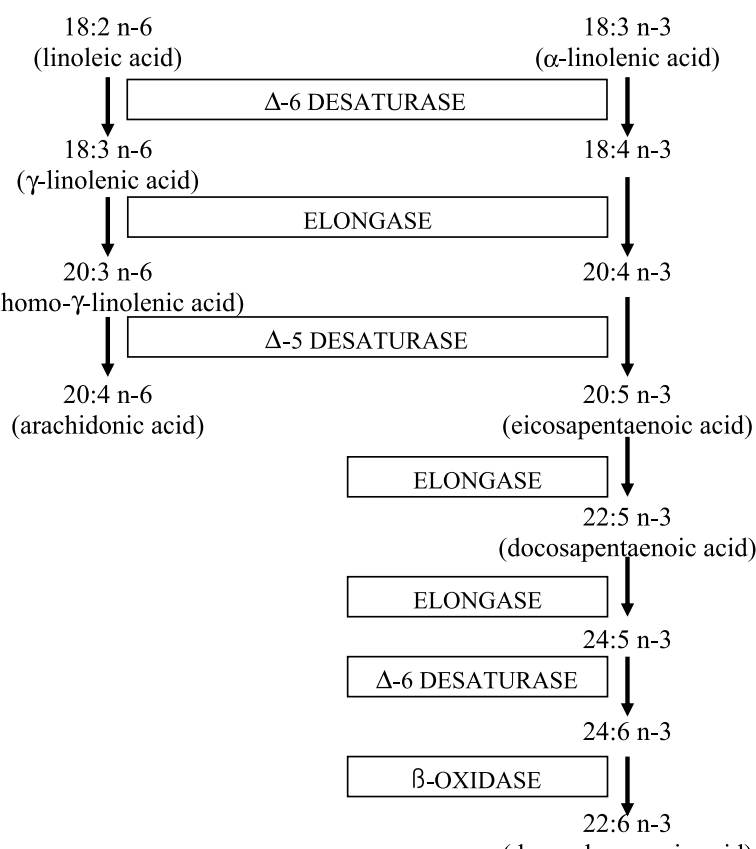

(docosahexaenoic acid)
Figure 1. Major metabolic pathways of n-3 and n-6 PUFA. 
18:3n- 6 were positively related to levels of 20:3n-6 and 20:4n-6; and levels of 20:3n-6 and 20:4n-6 were not correlated with each other. For n-3 fatty acids, levels of $18: 3 n-3$ were inversely related to the levels of 20:5n-3 and $22: 5 n-3$ and unrelated to 22:6n-3, whereas all long-chain n-3 fatty acids were positively correlated with each other. There were some correlations between $n-3$ and n- 6 fatty acids. Of note, 18:2n-6 and 18:3n-3 levels were positively correlated, and 20:4n-6 levels were positively related to all long-chain $n-3$ fatty acids but inversely related to 18:3n-3 levels. Trans fatty acid levels were positively related to $18: 3 n-3$ levels and inversely related to blood levels of 20:4n-6 and long-chain n-3 fatty acids (Table 2).

There was an inverse association between blood levels of $18: 2 n-6$ and overall prostate cancer risk (Table 3): Men in the highest quintile had a $38 \%$ lower risk of prostate cancer compared with men in the lowest quintile $\left(P_{\text {trend }}=0.03\right)$. Conversely, blood levels of $18: 3 n-6$ and $20: 3 n-6 s$, both metabolites of $18: 2 n-6$, were positively related to prostate cancer risk. The RR comparing extreme quintiles of these fatty acid levels was 1.41 [95\% confidence interval $(95 \% \mathrm{CI})$, $\left.0.94-2.12 ; P_{\text {trend }}=0.05\right]$ for $18: 3 n-6$ and $1.54(95 \%$ CI, $1.03-2.30$; $\left.P_{\text {trend }}=0.02\right)$ for $20: 3 n-6$. Blood levels of 20:4n-6 were unrelated to overall risk of prostate cancer. Among the n-3 fatty acids, blood levels of 18:3n-3 were unrelated to overall risk of prostate cancer, whereas levels of each of the long-chain n-3 fatty acids, as well as their sum, were associated with about $40 \%$ lower risk of prostate cancer. The strongest associations were for blood levels of 20:5n-3 and 22:5n-3 where men in the highest quintile of these fatty acids had a $43 \%(P=0.02)$ and $40 \%(P=0.01)$ lower risk of prostate cancer, respectively, when compared with men in the lowest quintile. Additional adjustment for fasting status, blood levels of trans fatty acids and the metabolic precursor fatty acids did not change these results (data not shown). Furthermore, there was no evidence that the associations between 20:4n-6, 20:5n-3, and 22:5n-3 and prostate cancer risk differed by fasting status $(P>0.05$ in all cases $)$.

Table 3. RR $(95 \% \mathrm{Cl})$ for prostate cancer by control quintiles of whole blood fatty acid levels

\begin{tabular}{|c|c|c|c|c|c|c|}
\hline & \multicolumn{5}{|c|}{ Quintile of fatty acid level } & \multirow[t]{2}{*}{$P_{\text {trend }}{ }^{*}$} \\
\hline & 1 (reference) & 2 & 3 & 4 & 5 & \\
\hline \multicolumn{7}{|l|}{ n-6 Fatty acids } \\
\hline \multicolumn{7}{|l|}{ Linoleic $(18: 2 n-6)$} \\
\hline Concentration, \% & 21.4 & 23.7 & 25.3 & 26.7 & 28.8 & \\
\hline Cases/controls & $121 / 96$ & $94 / 96$ & $99 / 96$ & $85 / 94$ & $77 / 94$ & \\
\hline Adjusted RR ${ }^{\top}$ & 1.00 & $0.73(0.49-1.11)$ & $0.77(0.52-1.16)$ & $0.68(0.45-1.03)$ & $0.62(0.41-0.95)$ & 0.03 \\
\hline \multicolumn{7}{|l|}{$\gamma$-Linolenic $(18: 3 n-6)$} \\
\hline Concentration, \% & 0.15 & 0.22 & 0.26 & 0.32 & 0.40 & \\
\hline Cases/controls & $88 / 94$ & $79 / 97$ & $94 / 95$ & $92 / 95$ & $123 / 95$ & \\
\hline Adjusted RR ${ }^{\top}$ & 1.00 & $0.87(0.57-1.33)$ & $1.05(0.69-1.60)$ & $1.04(0.68-1.57)$ & $1.41(0.94-2.12)$ & 0.05 \\
\hline \multicolumn{7}{|c|}{ Dihomo- $\gamma$-linolenic $(20: 3 n-6)$} \\
\hline Concentration, \% & 1.07 & 1.24 & 1.36 & 1.49 & 1.75 & \\
\hline Cases/controls & $83 / 94$ & $89 / 94$ & $81 / 95$ & $93 / 96$ & $130 / 97$ & \\
\hline Adjusted $\mathrm{RR}^{{ }^{\top}}$ & 1.00 & $1.06(0.70-1.60)$ & $0.97(0.63-1.49)$ & $1.11(0.74-1.66)$ & $1.54(1.03-2.30)$ & 0.02 \\
\hline \multicolumn{7}{|c|}{ Arachidonic (20:4n-6) } \\
\hline Concentration, \% & 7.9 & 9.3 & 10.1 & 10.9 & 12.3 & \\
\hline Cases/controls & $90 / 96$ & $110 / 96$ & $93 / 95$ & $86 / 94$ & $97 / 95$ & \\
\hline Adjusted $\operatorname{RR}^{\dagger}$ & 1.00 & $1.22(0.82-1.81)$ & $1.05(0.70-1.57)$ & $0.98(0.66-1.46)$ & $1.09(0.72-1.64)$ & 0.98 \\
\hline \multicolumn{7}{|l|}{ n-3 Fatty acids } \\
\hline \multicolumn{7}{|l|}{$\alpha$-Linolenic $(18: 3 n-3)$} \\
\hline Concentration, $\%$ & 0.24 & 0.30 & 0.35 & 0.41 & 0.54 & \\
\hline Cases/controls & $83 / 97$ & $97 / 96$ & $98 / 94$ & $90 / 95$ & $108 / 94$ & \\
\hline Adjusted RR ${ }^{\top}$ & 1.00 & $1.17(0.78-1.75)$ & $1.19(0.81-1.76)$ & $1.10(0.73-1.65)$ & $1.31(0.89-1.95)$ & 0.24 \\
\hline \multicolumn{7}{|c|}{ Eicosapentaenoic (20:5n-3) } \\
\hline Concentration, $\%$ & 1.28 & 1.58 & 1.79 & 1.99 & 2.36 & \\
\hline Cases/controls & $109 / 95$ & $106 / 96$ & $96 / 95$ & $96 / 97$ & $69 / 93$ & \\
\hline Adjusted RR ${ }^{\top}$ & 1.00 & $0.93(0.62-1.38)$ & $0.83(0.54-1.25)$ & $0.77(0.49-1.21)$ & $0.57(0.36-0.92)$ & 0.02 \\
\hline \multicolumn{7}{|c|}{ Docosapentaenoic (22:5n-3) } \\
\hline Concentration, \% & 0.77 & 0.88 & 0.97 & 1.06 & 1.19 & \\
\hline Cases/controls & $114 / 96$ & $106 / 94$ & $103 / 96$ & $79 / 94$ & $74 / 96$ & \\
\hline Adjusted RR ${ }^{\top}$ & 1.00 & $0.93(0.63-1.37)$ & $0.88(0.59-1.30)$ & $0.68(0.45-1.02)$ & $0.60(0.38-0.93)$ & 0.01 \\
\hline \multicolumn{7}{|c|}{ Docosahexaenoic (22:6n-3) } \\
\hline Concentration, \% & 1.42 & 1.89 & 2.19 & 2.65 & 3.37 & \\
\hline Cases/controls & $100 / 95$ & $82 / 94$ & $119 / 95$ & $116 / 97$ & $59 / 95$ & \\
\hline Adjusted RR & 1.00 & $0.85(0.57-1.28)$ & $1.22(0.81-1.84)$ & $1.14(0.76-1.71)$ & $0.60(0.39-0.93)$ & 0.07 \\
\hline \multicolumn{7}{|l|}{ Long-chain $n-3 \neq$} \\
\hline Concentration, \% & 3.66 & 4.44 & 4.94 & 5.57 & 6.70 & \\
\hline Cases/controls & $107 / 95$ & $105 / 95$ & $112 / 94$ & $84 / 98$ & $68 / 94$ & \\
\hline Adjusted $\mathrm{RR}^{\dagger}$ & 1.00 & $0.97(0.66-1.44)$ & $1.02(0.67-1.54)$ & $0.74(0.49-1.13)$ & $0.59(0.38-0.93)$ & 0.01 \\
\hline \multicolumn{7}{|l|}{$\mathrm{n}-6 / \mathrm{n}-3$ ratios } \\
\hline \multicolumn{7}{|l|}{ Linoleic/ $\alpha$-linolenic } \\
\hline Ratio & 45.8 & 62.0 & 70.8 & 82.8 & 103.7 & \\
\hline Cases/controls & $111 / 96$ & $107 / 93$ & $99 / 94$ & $71 / 97$ & $88 / 96$ & \\
\hline Adjusted $\mathrm{RR}^{\dagger}$ & 1.00 & $1.01(0.67-1.52)$ & $0.91(0.62-1.33)$ & $0.61(0.40-0.94)$ & $0.80(0.54-1.19)$ & 0.08 \\
\hline \multicolumn{7}{|c|}{ Arachidonic/eicosapentaenoic } \\
\hline Ratio & 4.18 & 5.08 & 5.66 & 6.42 & 7.64 & \\
\hline Cases/controls & $80 / 94$ & $86 / 97$ & $99 / 95$ & $116 / 96$ & $95 / 94$ & \\
\hline Adjusted $\mathrm{RR}^{\dagger}$ & 1.00 & $1.06(0.69-1.63)$ & $1.26(0.83-1.91)$ & $1.52(0.98-2.37)$ & $1.30(0.80-2.11)$ & 0.14 \\
\hline
\end{tabular}


Table 4. Adjusted RR of prostate cancer comparing top to bottom quintiles of blood fatty acid levels according to tumor characteristics

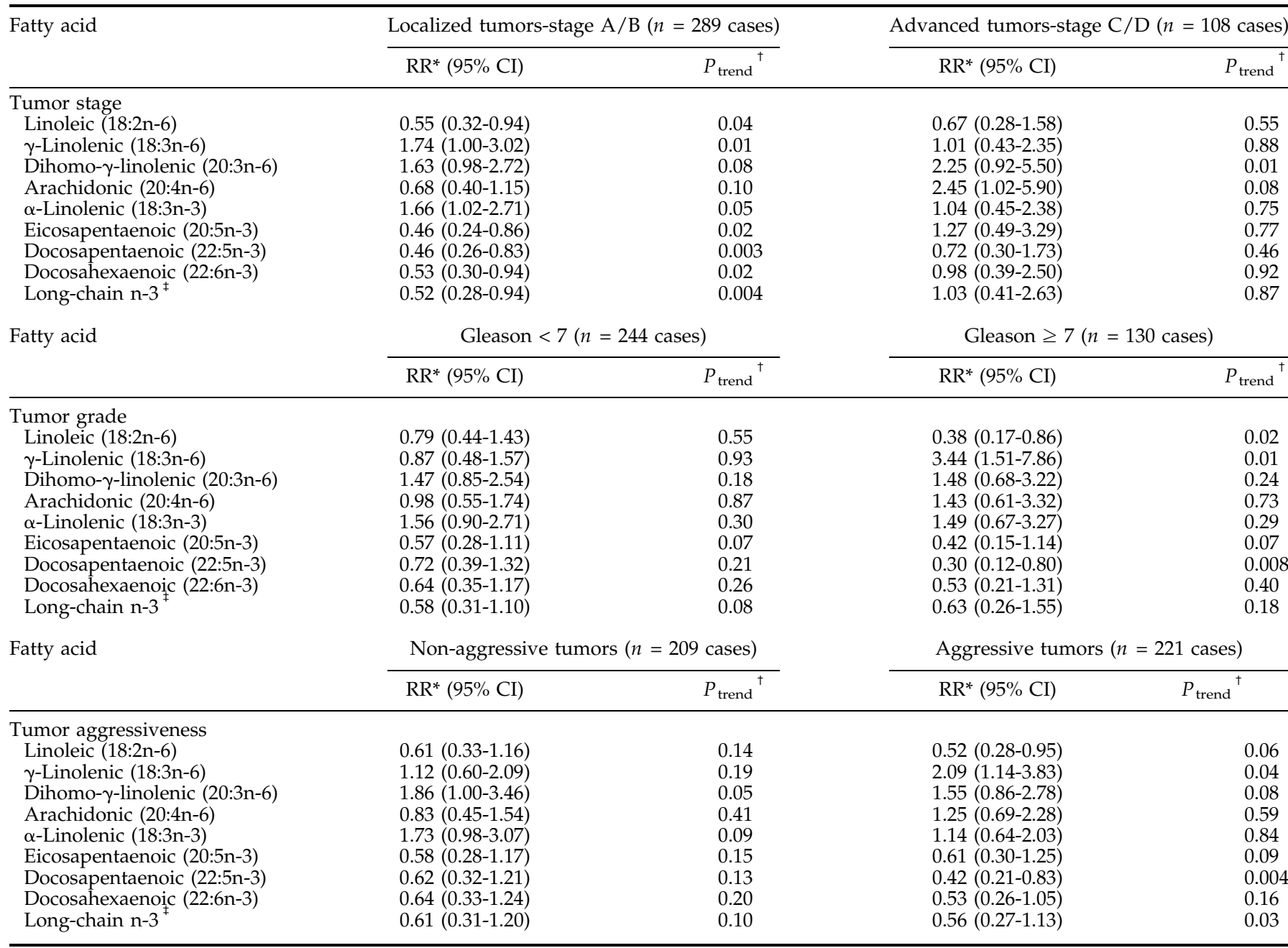

NOTE: Adjusted for matching factors (age, smoking status at baseline, and length of follow-up).

*For men in the highest quintile of the specific fatty acid in comparison to men in the lowest quintile.

tCalculated with median fatty acid concentration in each quintile as a continuous variable.

$\ddagger$ Eicosapentaenoic acid + docosapentaenoic acid + docosahexaenoic acid.

We assessed whether the ratio of n-6 to n-3 fatty acids of the same carbon chain length that might compete for key enzymes in their metabolic pathways (Fig. 1) was related to prostate cancer risk (Table 3). Men in the fourth quintile of the ratio of $18: 2 n-6$ to $18: 3 n-3$ levels in blood had a $39 \%$ lower risk of prostate cancer than men in the lowest quintile $(P=0.02)$. However, tests of interaction as to whether the association between 18:3n-3 and prostate cancer might differ by levels of $18: 2 \mathrm{n}-6$ and vice versa were not significant $\left(P_{\text {interaction }}=0.17\right.$ and 0.55 , respectively). The ratio of $20: 4 n-6$ to $20: 5 n-3$ levels in blood was unrelated to prostate cancer risk. Tests for interaction as to whether the association between 20:4n-6 and prostate cancer might differ by levels of $20: 5 n-3$ and vice versa were not significant either $\left(P_{\text {interaction }}=0.61\right.$ and 0.34 , respectively). We also found no evidence of interaction between aspirin assignment and blood levels of long-chain PUFA (20:4n-6, 20:5n-3, 22:5n-3, and 22:6n-3) in relation to prostate cancer risk.

To assess whether blood fatty acid levels might be influenced by preclinical cancer, we then divided the analyses by date of diagnosis. Blood levels of 18:2n-6 and all the long-chain n-3 fatty acids were related to prostate cancer cases occurring later in the follow-up but not to cases occurring during the first 8 years of the study, whereas the converse was true for blood levels of 18:3n-6 and 20:3n-6. The RR $\mathrm{R}_{\mathrm{Qvs} . \mathrm{Q} 1}$ of prostate cancer occurring early and late during follow-up were $0.53(95 \% \mathrm{CI}$, $\left.0.26-1.10 ; P_{\text {trend }}=0.20\right)$ and $0.61\left(95 \%\right.$ CI, $\left.0.37-1.03 ; P_{\text {trend }}=0.04\right)$ for $18: 2 \mathrm{n}-6,0.80\left(95 \% \mathrm{CI}, 0.36-1.79 ; P_{\text {trend }}=0.42\right)$ and $0.52(95 \%$ CI, $\left.0.30-0.91 ; P_{\text {trend }}=0.008\right)$ for total long-chain $\mathrm{n}-3,1.66(95 \%$ CI, $\left.0.75-3.71 ; P_{\text {trend }}=0.06\right)$ and $1.40\left(95 \%\right.$ CI, 0.86-2.28; $P_{\text {trend }}=$ $0.23)$ for $18: 3 n-6$, and $2.09\left(95 \%\right.$ CI, $\left.0.98-4.49 ; P_{\text {trend }}=0.04\right)$ and 1.37 (95\% CI, 0.85-2.21; $\left.P_{\text {trend }}=0.15\right)$ for $20: 3 n-6$.

Lastly, we examined whether the associations between blood levels of individual PUFA differed by tumor stage, grade, or clinical aggressiveness (Table 4). Blood levels of 18:2n-6 and 18:3n-6 seemed to be stronger for localized, highgrade tumors with a clinically aggressive behavior. The association of 18:2n-6 with high-grade and aggressive tumors became stronger after adjustment for 18:3n-3 and trans fatty acids (data not shown). In contrast, blood levels of 20:3-6 were positively associated to prostate cancer risk regardless of tumor stage, grade, or aggressiveness. Blood levels of 20:4n-6 were positively related to advanced tumors. This association became nonsignificant after adjustment for trans fatty acids $\left(P_{\text {trend }}=0.16\right)$. Among the $\mathrm{n}-3$ fatty acids, there was a positive association between 18:3n-3 levels in blood and risk of localized tumors that disappeared after adjusting for levels of trans fatty acids $\left(P_{\text {trend }}=0.11\right)$. Blood levels long-chain $n-3$ 
fatty acids, particularly of 20:5n-3 and 22:5n-3, seemed to be more strongly related to a lower risk of localized, high-grade tumors with a clinically aggressive behavior. The associations of n-3 long-chain fatty acids with high-grade and aggressive tumors became stronger after adjustment for 18:2n-6, 18:3n-3, and trans fatty acids (data not shown). Formally testing whether the associations between these fatty acids and prostate cancer differed by tumor aggressiveness, however, did not reveal statistically significant differences $(P>0.05$ in all cases).

\section{Discussion}

In this prospective examination, we found that higher levels of long-chain n-3 fatty acids and 18:2n-6 were associated with a decreased risk of prostate cancer, whereas higher levels of some fatty acids resulting from the desaturation and elongation of 18:2n-6 were associated with an increased risk of prostate cancer. For 18:2n-6 and long-chain n-3 fatty acids, these associations were present even among cases diagnosed 8 to 15 years after the blood samples were collected. Furthermore, blood levels of 18:2n-6, 20:5n-3, and 22:5n-3s seemed to be more strongly related to clinically aggressive tumors.

Levels of PUFA in whole blood are suitable biomarkers of fatty acid intake, in particular for fatty acids that are not endogenously synthesized (11). Baylin et al. found reasonably high correlations between dietary intake of several fatty acids and their levels in whole blood. The correlations were 0.43 for $18: 2 n-6,0.38$ for $18: 3 n-3,0.22$ for $20: 5 n-3$, and 0.23 for $22: 6 n-3$ (11). However, blood levels of 18:3n-6, 20:3n-6, and 20:4n-6 were unrelated to dietary intake of these fatty acids, and the correlation between 18:2n-6 intake and blood levels of 20:4n-6 was negligible (11). Therefore, in interpreting our results, blood levels of 18:2n- 6 and the n- 3 fatty acids examined can be regarded as markers of intake, whereas levels of the remaining n-6 fatty acids are likely to represent the activity of enzymes participating in the desaturation and elongation of $18: 2 n-6$, not diet. Whole blood fatty acid levels are highly correlated with plasma and adipose tissue levels of the corresponding fatty acid (11). Thus, our results can be readily compared with previous studies using other biological specimens as biomarkers of fatty acid intake.

Some of our findings, in particular the inverse relation between 18:2n-6 levels and prostate cancer risk, in contrast to what would be expected from animal and in vitro studies. In general, these studies have found that 18:2n-6 promotes prostate cancer growth $(3,13-15)$. However, results from epidemiologic studies do not support the notion that 18:2n-6 intake increases prostate cancer risk $(16,17)$. Most investigations have yielded null findings $(5,16,18-25)$, with fewer studies reporting statistically significant positive $(26,27)$ or inverse associations $(4,28,29)$ between 18:2n-6 and prostate cancer risk. In previous studies where tissue levels of 18:2n-6 have been related to prostate cancer risk, those where tissues were collected after cancer diagnosis have reported positive associations with prostate cancer $(26,27)$, whereas prospective studies have yielded inverse associations $(4,5,19)$, albeit not always statistically significant. This difference should be carefully considered because some authors have documented altered metabolism of other n- 6 fatty acids in prostate cancer tissue (30), and whether prostate cancer causes systemic alterations in n-6 fatty acid metabolism is unknown. This should also be considered as a potential explanation for our findings regarding 18:3n-6 and 20:3n-6 as blood levels of these fatty acids were only associated with prostate cancer risk during early years of follow-up.

We found that blood 18:2n- 6 levels were inversely related to the risk of aggressive tumors. This result is in agreement with our previous report of a weak (although not statistically significant) inverse relation between plasma 18:2n-6 levels and prostate cancer risk (5), given that in that study, most of the cases had advanced disease at diagnosis. Only two prospective studies, both using food frequency questionnaires to assess $18: 2 n-6$ intake, have explored this association separately for organ confined and advanced tumors; and in both cases, these associations were null $(18,20)$. Neither of the previous prospective studies using biomarkers of 18:2n-6 intake reported their results separately by tumor aggressiveness, but their findings are consistent with ours $(4,19)$.

Another finding that is apparently not consistent with animal and in vitro studies is that for 18:3n-3 that we found not to be associated with prostate cancer risk overall and when examined by most tumor characteristics. The exception was a positive association with localized tumors that disappeared after adjustment for trans fatty acids. It has traditionally been assumed that 18:3n-3 would decrease prostate cancer risk. This idea is based on the observation that long-chain n- 3 fatty acids inhibit prostate cancer proliferation $(3,14)$ and the fact that long-chain n-3 fatty acids can be metabolically derived from $18: 3 n-3$ (31). However, several studies have shown that the conversion of $18: 3 n-3$ into long-chain $n-3$ is minimal (32-35). In addition, animal studies that have evaluated the role of 18:3n-3 on prostate carcinogenesis have failed to document antiproliferative effects (14) and, in some cases, have actually documented tumor promoting effects (15). Our results contrast with those based on the first 6 years of follow-up from this cohort (5) and with a growing body of literature suggesting a positive association between 18:3n-3 intake and risk of prostate cancer overall $(19,21,25,27)$ and clinically aggressive tumors in particular (18). However, only one of these studies has considered the potential of confounding by trans fatty acids (18) that share common food sources with 18:3n-3 and can inhibit the activity of the enzymes metabolizing this fatty acid.

Levels of the three long-chain n-3 fatty acids examined were inversely related with reduced risk of prostate cancer overall and seemed to be more strongly related with localized, highgrade, aggressive tumors. The strongest association observed was that of 22:5n-3 and aggressive and high-grade prostate tumors. Long-chain n-3 fatty acids share common food sources, mainly fish and seafood (36). In addition, 22:5n-3 is a product of the conversion of $20: 5 n-3$ into $22: 6 n-3$ and of retroconversion in the same metabolic pathway (31). Thus, our results are consistent with previous studies of prostate cancer and long-chain n-3 fatty acids (mainly 20:5n-3 and 22:6n-3) or fish intake $(18,25,37-40)$, although several others found no association $(4,5,19,20,26,27)$. Our findings are also in agreement with various animal models of prostate cancer, which suggest tumor growth inhibition by long-chain n-3 fatty acids $(3,14,15,41)$. Although elucidating whether there are different cancer preventive benefits of each of these fatty acids may be of scientific interest, it may not have important practical implications due the considerations presented above.

High levels of 18:3n-6 were associated with an increased risk of aggressive prostate cancer, whereas high levels of 20:3n-6 were more strongly related to non-aggressive tumors. We are not aware of previous studies examining the association between levels of these fatty acids and prostate cancer risk in humans. We did not observe an association between blood levels of 20:4n-6 and prostate cancer overall or by tumor characteristics, in agreement with previous epidemiologic studies $(5,19,25,27)$. Because whole blood levels of 18:3n-6, 20:3n-6, and 20:4n-6 are minimally dependent on diet, future studies could explore whether genetic variation in the enzymes involved in this metabolic pathway may affect differences in tissue levels of the resulting fatty acids and prostate cancer risk.

Our study has several strengths. First, blood samples were collected before prostate cancer diagnosis, in some cases, 
several years in advance. In addition, the associations of 18:2n6 and n-3 fatty acids with prostate cancer observed among the entire study group were also present among men diagnosed 8 to 15 years after blood was collected. Thus, the possibility that metabolic disturbances caused by disease affected these results is minimal in our study. The prospective design and high follow-up rates of the study decrease the possibility that our findings could be result of a bias. The large number of cases allowed us to examine these associations with greater statistical power than had been possible before for total, nonaggressive, and aggressive prostate cancer. The most important limitation of our study is the possibility that residual and unmeasured factor associated with blood fatty acid levels may be responsible for the observed associations because our study is observational. Nevertheless, we evaluated a number of variables as potential confounders and found that adjusting for the few variables associated with fatty acid levels and prostate cancer had minimal effect on most of the results. Although multiple statistical tests could be another potential limitation of this study, all these analyses were based on our priori hypotheses and therefore biologically justifiable. Lastly, although our study was adequately powered to detect moderate associations with overall prostate cancer, larger studies will be required to evaluate whether the associations between specific fatty acid levels in blood and prostate cancer differ by tumor characteristics as our findings suggest but could not confirm.

In summary, the relationship between PUFA and prostate cancer may be more complex than that suggested by animal models. Our data suggest that intake of PUFA is unlikely to increase prostate cancer risk, and some of them, particularly linoleic and long-chain n-3 fatty acids, may actually decrease the risk of developing clinically aggressive prostate tumors. Because intake of polyunsaturated fats may help prevent other common chronic diseases, notably heart disease and diabetes (42-44), our findings, if confirmed by other studies, may have a broader implication in chronic disease prevention.

\section{References}

1. American Cancer Society. Cancer facts and figures 2005. Atlanta: American Cancer Society, 2005

2. Chan JM, Gann PH, Giovannucci EL. Role of diet in prostate cancer development and progression. J Clin Oncol 2005;23:8152-60.

3. Rose D. Effects of dietary fatty acids on breast and prostate cancers: evidence from in vitro experiments and animal studies. Am J Clin Nutr 1997;66:1513-22S.

4. Laaksonen DE, Laukkanen JA, Niskanen L, et al. Serum linoleic and total polyunsaturated fatty acids in relation to prostate and other cancers: a population-based cohort study. Int J Cancer 2004;111:444-50.

5. Gann PH, Hennekens CH, Sacks FM, et al. Prospective study of plasma fatty acids and risk of prostate cancer. J Natl Cancer Inst 1994;86:281-6.

6. Steering Committee of the Physicians' Health Study Research Group. Final report on the aspirin component of the ongoing Physicians' Health Study. N Engl J Med 1989;321:129-35.

7. Hennekens $\mathrm{CH}$, Buring JE, Manson JE, et al. Lack of effect of long-term supplementation with beta carotene on the incidence of malignant neoplasms and cardiovascular disease. N Engl J Med 1996;334:1145-9.

8. Catalona WI, Avioli LV. Diagnosis, staging, and surgical treatment of prostatic carcinoma [clinical conference]. Arch Intern Med 1987;147:361-3.

9. Zock PL, Gerristen J, Katan M. Partial conservation of the sn-2 position of dietary triglicerides in fasting plasma lipids in humans. Eur J Clin Invest 1996;26:141-50.

10. Zock PL, Mensink RP, Harryvan J, de Vries JH, Katan M. Fatty acids in serum cholesteryl esters as quantitative biomarkers of dietary intake in humans. Am J Epidemiol 1997;145:1114-22.

11. Baylin A, Kim MK, Donovan-Palmer A, et al. Fasting whole blood as a biomarker of essential fatty acid intake in epidemiologic studies: comparison with adipose tissue and plasma. Am J Epidemiol 2005;162:373-81.

12. Zeleniuch-Jacquotte A, Chajes V, Van Kappel AL, Riboli E, Toniolo P. Reliability of fatty acid composition in human serum phospholipids. Eur J Clin Nutr 2000;54:367-72.

13. Wang Y, Corr JG, Thaler HT, et al. Decreased growth of established human prostate LNCaP tumors in nude mice fed a low-fat diet. J Natl Cancer Inst 1995;87:1456-62
14. Connolly JM, Coleman M, Rose DP. Effects of dietary fatty acids on DU145 human prostate cancer cell growth in athymic nude mice. Nutr Cancer 1997; 29:114-9.

15. Pandalai PK, Pilat MJ, Yamasaki K, Naik H, Pienta KJ. The effects of omega3 and omega- 6 fatty acids on in vitro prostate cancer growth. Anticancer Res 1996;16:815-20.

16. Zock PL, Katan MB. Linoleic acid intake and cancer risk: a review and metaanalysis. Am J Clin Nutr 1998;68:142-53.

17. Willett WC. Specific fatty acids and risks of breast and prostate cancer: dietary intake. Am J Clin Nutr 1997;66:1557-63s.

18. Leitzmann MF, Stampfer MJ, Michaud DS, et al. Dietary intake of n-3 and n-6 fatty acids and the risk of prostate cancer. Am J Clin Nutr 2004;80: 204-16.

19. Harvei S, Bjerve KS, Tretli S, et al. Prediagnostic level of fatty acids in serum phospholipids: omega- 3 and omega- 6 fatty acids and the risk of prostate cancer. Int J Cancer 1997;71:545-51.

20. Schuurman AG, van den Brandt PA, Dorant E, Brants HAM, Goldbohm RA. Association of energy and fat intake with prostate carcinoma risk. Cancer 1999;86:1019-27.

21. De Stefani E, Deneo-Pellegrini H, Boffetta P, Ronco A, Mendilaharsu M a-Linolenic acid and risk of prostate cancer: a case-control study in Uruguay. Cancer Epidemiol Biomarkers Prev 2000;9:335-8.

22. Anderson SO, Wolk A, Bergström R, et al. Energy, nutrient intake and prostate cancer risk: a population-based case-control study in Sweden. Int J Cancer 1996;68:716-22.

23. Bairaty I, Meyer F, Fradet Y, Moore L. Dietary fat and advanced prostate cancer. J Urol 1998;159:1271-5.

24. Meyer F, Bairaty I, Fradet Y, Moore L. Dietary energy and nutrients in relation to preclinical prostate cancer. Nutr Cancer 1997;29:120-6.

25. Yang YJ, Lee SH, Hong SJ, Chung BC. Comparison of fatty acid profiles in the serum of patients with prostate cancer and benign prostatic hyperplasia. Clin Biochem 1999;32:405-9.

26. Godley P, Campbell M, Gallagher P, et al. Biomarkers of essential fatty acid consumption and risk of prostatic carcinoma. Cancer Epidemiol Biomarkers Prev 1996;5:889-95.

27. Newcomer LM, King IB, Wicklund KG, Stanford JL. The association of fatty acids with prostate cancer risk. Prostate 2001;47:262-8.

28. Kaul L, Heshmat MY, Kov IJ, et al. The role of diet in prostate cancer. Nutr Cancer 1987;9:123-8.

29. Bidoli E, Talamini R, Bossetti $C$, et al. Macronutrients, fatty acids, cholesterol and prostate cancer risk. Ann Oncol 2005;16:152-7.

30. Chaudry AA, Wahle KWJ, McClinton S, Moffat LEF. Arachidonic acid metabolism in benign and malignant prostatic tissue in vitro: effects of fatty acids and cyclooxygenase inhibitors. Int J Cancer 1994;57:176-80.

31. Rose DP, Connolly JM. Omega-3 fatty acids as cancer chemopreventive agents. Pharmacol Ther 1999;83:217-44

32. Emken EA, Adlof RO, Gulley RM. Dietary linoleic acid influences desaturation and acylation of deuterium-labeled linoleic and linolenic acids in young adult males. BBA-Lipid Lipid Metab 1994;1213:277-88.

33. Gerster H. Can adults adequately covnert a-linolenic (18:3n-3) to eicosapentaenoic (20:5n-3) and docosahexaenoic acid (22:6n-3)? Int J Vitam Nutr Res 1998;68:159-73.

34. Burdge GC, Finnegan YE, Minihane AM, Williams CM, Wotton SA. Effect of altered dietary n-3 fatty acid intake upon plasma lipid fatty acds composition, conversion of [13C]a-linolenic acids to longer-chain fatty acids and partitioning towards b-oxidation in older men. Br J Nutr 2003; 90:311-21.

35. Hussein N, Ah-Sing E, Wilkinson $\mathrm{P}$, et al. Long-chain conversion of [13C]linoleic acid and \{alpha\}-linolenic acid in response to marked changes in their dietary intake in men. J Lipid Res 2005:46:269-80.

36. Meyer BJ, Mann NJ, Lewis JL, et al. Dietary intakes and food sources of omega-6 and omega-3 polyunsaturated fatty acids. Lipids 2003;38 $391-8$.

37. Augustsson K, Michaud DS, Rimm EB, et al. A prospective study of intake of fish and marine fatty acids and prostate cancer. Cancer Epidemiol Biomarkers Prev 2003;12:64-7.

38. Terry P, Lichtenstein P, Feychting M, Ahlbom A, Wolk A. Fatty fish consumption and risk of prostate cancer. Lancet 2001;357:1764-6.

39. Pawlega J, Rachtan J, Dyba T. Dietary factors and risk of prostate cancer in Poland. Results of case-control study. Neoplasma 1996;43:61-3.

40. Norrish AE, Skeaff CM, Arribas GL, Sharpe SJ, Jackson RT. Prostate cancer risk and consumption of fish oils: a dietary biomarker-based case-control study. Br J Cancer 1999;81:1238-42.

41. Karmali RA, Reichel $P$, Cohen LA, et al. The effects of dietary omega- 3 fatty acids on the DU-145 transplantable human prostatic tumor. Anticancer Res 1987:7:1173-80.

42. Ascherio A, Rimm EB, Giovannucci EL, et al. Dietary fat and risk of coronary heart disease in men: cohort follow up study in the United States. Br Med J 1996;313:84-90.

43. Mozaffarian D, Ascherio A, Hu FB, et al. Interplay between different polyunsaturated fatty acids and risk of coronary heart disease in men. Circulation 2005;111:157-64.

44. van Dam RM, Willett WC, Rimm EB, Stampfer MJ, Hu FB. Dietary fat and meat intake in relation to risk of type 2 diabetes in men. Diabetes Care 2002; 25:417-24. 


\section{Cancer Epidemiology, \\ Biomarkers \& Prevention}

\section{A Prospective Study of Polyunsaturated Fatty Acid Levels in Blood and Prostate Cancer Risk}

Jorge E. Chavarro, Meir J. Stampfer, Haojie Li, et al.

Cancer Epidemiol Biomarkers Prev 2007;16:1364-1370.

Updated version Access the most recent version of this article at:

http://cebp.aacrjournals.org/content/16/7/1364

Cited articles This article cites 43 articles, 10 of which you can access for free at:

http://cebp.aacrjournals.org/content/16/7/1364.full\#ref-list-1

Citing articles This article has been cited by 15 HighWire-hosted articles. Access the articles at:

http://cebp.aacrjournals.org/content/16/7/1364.full\#related-urls

E-mail alerts Sign up to receive free email-alerts related to this article or journal.

Reprints and To order reprints of this article or to subscribe to the journal, contact the AACR Publications

Subscriptions Department at pubs@aacr.org.

Permissions To request permission to re-use all or part of this article, use this link http://cebp.aacrjournals.org/content/16/7/1364.

Click on "Request Permissions" which will take you to the Copyright Clearance Center's (CCC)

Rightslink site. 